\title{
A contradiction-driven approach to theory formation: Conceptual issues, Pragmatics in human learning, potentialities
}

\author{
Germana Menezes da Nóbrega \\ Mestrado em Gestão do Conhecimento e da \\ Tecnologia da Informação \\ Universidade Católica de Brasília (UCB) \\ SGAN 916 - Módulo B - Asa Norte \\ 70190-045 - Brasília - DF - Brazil \\ gmnobrega@pos.ucb.br
}

\author{
Stefano A. Cerri2, Jean Sallantin \\ Laboratoire d'Informatique, de Robotique et de \\ Micro-électronique de Montpellier (LIRMM) \\ UMR 5506 - Université Montpellier II/CNRS \\ 161, Rue Ada, 34392 \\ Montpellier cedex 5, France \\ \{cerri,sallantin\}@lirmm.fr
}

\begin{abstract}
.
In Educational literature, Discovery Learning appears as an approach in which the learner builds up his/her own knowledge by performing experiments within a domain and inferring/increasing rules as a result. Such a constructivist approach has been largely exploited in the design of computational artefacts with learning purposes, the so-called Discovery Learning Environments (DLEs). One known feature of such environments is the autonomy degree required for students to succeed while handling a domain. Additionally, DLEs designers are often challenged to get students actually engaged. Such questions are on the basis of our concerns with the design and usage of particular DLEs, within which learning events occur as a consequence of contradiction detection and overcoming, during human/machine cooperative work. In this paper, we present an artificial agent capable of handling such a contradiction-driven approach of learning, by highlighting the exchanges that the agent should promote with a human learner. The conceptual model supporting the agent's design relies on the scientific rationale, particularly the empirical approach guided by the theory-experiment confrontation. We shall reinforce the interest of the model for the design of DLEs by presenting its exploitation in a real learning situation in Law. Also, we suggest potential instantiations of the model elsewhere than in Human Learning.
\end{abstract}

Key words: Theory formation, Discovery Learning, Contradiction, Human/Computer Cooperation.

\section{Introduction}

The model of knowledge formalizing known as Phicalculus [16] has appeared as an attempt to abstract a successful approach practiced since 1994 by the lawyers of the Company Fidal-KPMG grouping 1200 lawyers in France. Lawyer's daily activity consists of understanding, proving and comparing contracts. The issue for innovation for them is that laws and norms change continuously, so contracts have to be conceived accordingly. The Company has identified classes of contracts, and for each class has decided to offer lawyers a Contractual Framework (CF). A CF represents the knowledge that enables the artificial agent fid@ct to assist the lawyers in their activity. Whenever the Company identifies a contract class, it delegates the design of the corresponding $\mathrm{CF}$ to a team composed of a senior lawyer and two novice lawyers, who are supposed to interact both with each other and with fid@ct aiming a CF.

Fidal's method to design a CF may be summarized as follows (Figure 1): after having analyzed a number of contracts previously written by experimented lawyers of the Company, the novice lawyers propose a $\mathrm{CF}$, and then test and revise it under 


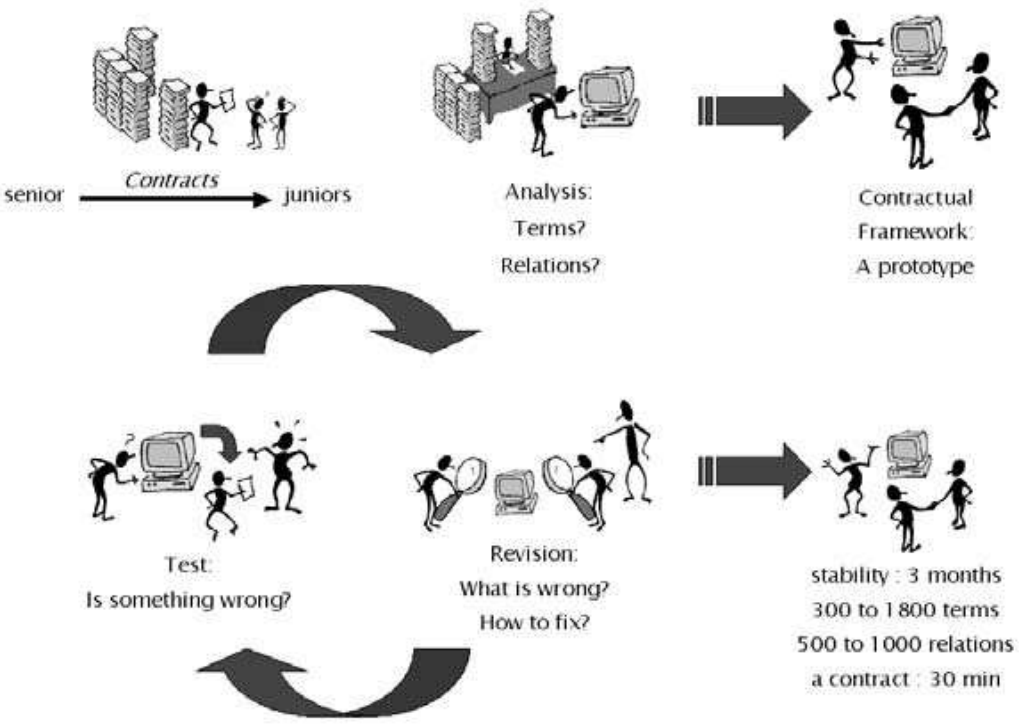

Figure 1: Scenario of Fidal's method

the supervision of the senior lawyer. The cycle is repeated until a CF is judged stable by the senior. CFs thus constructed are used by fid@ct to assist about 400 lawyers of the Company, and it takes about 30 minutes instead of several hours, for a lawyer to write a contract assisted by the agent. The Company has patented both the method and the agent fid@ct.

While a Contractual Framework itself is the object of main interest for Fidal's lawyers, from the perspective of Human Learning, we are mainly interested in its process of construction. The main question we address is: do novice lawyers learn something due to the interaction both with the senior lawyer and the fid@ct agent during the process of construction of a CF? In a previous paper [14], we argue on a characterization of a Learning Environment (LE) for Fidal's method. As a starting point, we adopt as a principle the view of Human Learning as a potential, indirect side effect of Dialogues, the agreement resulting from discussions by eminent scholars, as summarized in [8].

A popular property of LEs is that they often embody some "true" knowledge that is supposed to be "acquired" by the learner by interacting with the system. Socratic tutoring methods, on the contrary, attempt to emulate the autonomous discovery process for the causes of inconsistencies by the learner as a consequence of challenging him/her with dialectic arguments. In this direction, "the concept of discovery learning has appeared numerous times throughout history as a part of the educational philosophy of many great philosophers particularly Rousseau, Pestalozzi and Dewey, 'there is an intimate and necessary relation between the process of actual experience and education [17]. It also enjoys the support of learning theorists/psychologists Piaget, Bruner, and Papert, 'Insofar as possible, a method of instruction should have the objective of leading the child to discover for himself' [6,3]. In spite of these pedagogical suggestions, few LEs are founded on these principles. Rather, most Les developers wish "the truth in a domain" to be acquired by learners exposed to "the truth". In Fidal's method, in order to propose a CF, novice lawyers perform abstraction by themselves as they analyze achieved contracts in order to establish what terms should appear in a Contractual Framework for the corresponding contract class. Also, they infer autonomously logical constraints stressing relations among those terms, in a meaningful manner. In Human Learning, as stated by [7], building meaningful relations should reflect the student's understanding of a domain, being a harder task than coming up with the concepts themselves. Moreover, within fid@act, the prominent view that the "true" knowledge should be in the machine is changed, since it is provided only with the capability of handling propositional constraints among terms. Such a capability allows the agent to work like a mirror, reflecting thus to the novices inconsistencies in the knowledge that they have externalized during the process.

Discovery learning is also characterized as an iterative approach, in which errors work like a source of revision, as knowledge is supposed to be constructed by trial and error from experience. This issue is also present in the rationale of Fidal's lawyers: inconsistencies detected are exploited aiming to improve a CF. The convergence, often a problem with this kind of approach, is achieved thanks to the fact that knowledge represented by RFs 
reflect the way of thinking and working of a group, and according to the Law to which they are submitted. This meets the constructivist views of learning: knowledge/learning are depending on context, person, and social situation [21].

Recent work [2] on EIAO privilege methods and tools facilitating the acquisition of meta-cognitive or soft skills, with respect to domain-independent "true" knowledge and skills. We see the development of cognitive skills as a potential consequence of discovery learning activities, since explanation and argumentation capabilities are crucial to perform tasks like build up a hypothesis or interpret experimental results such as required to revise or confirm hypotheses. Soft skills are also a requirement whenever the control is transferred to the learner interacting with a system [1]. In Fidal's method these two (in principle controversing) perspectives co-exist and are even complementary since individuals are embedded in a collaborative environment. Collaborative work has shown its relevance both to learning [18,5] and in a widespread context [4]. In addition, recent work on ITS [44] points out to how discovery learning and collaborative learning may be brought together in order to design effective LE's.

The dialectic, autonomous, domain-independent, constructivist, and collaborative aspects of Fidal's approach suggest us a positive answer to the question that we are addressing (do novices lawyers learn anything...?). Expecting to confirm our assumption (that the answer is positive), we have decided to expose real learners to the method. In order to support the learner's work, we have implemented a Webyserved LE, called PhiInEd [15,13]. From a widespread perspective, the work on PhiInEd can be seen as an attempt to validate the model Phi-calculus when this latter meets the cause of Human Learning.

In $\S 2$ we partially present Phi-calculus as a model for the design of DLEs; these could be thought of as artificial agents supposed to stimulate learning by handling contradictions, while interacting with a human learner. In $\$ 3$ we focus on structural and dynamics components of the model itself. Then, in $\S 4$ , a Web-served Learning Environment is presented as a particular instantiation of the model. In $\$ 5$ we report a real study situation held upon that Web-server. In $\$ 6$ we point out to Phi-calculus potential exploitations beyond Human Learning. Finally, in $\$ 7$ we present our concluding remarks and highlight ongoing work.

\section{Phi-calculus: a contradiction-driven and computer-aided human learning model}

In order to present Phi-calculus as a model for the design of DLEs we shall consider:

- An Artificial Agent provided with no initial knowledge, but having two skills, namely, (i) handling propositional logical constraints, and (ii) learning from examples [34,33];

- An illustrating scenario developed upon a classical toy domain, which has been chosen in order to facilitate the presentation of both structural and functional elements of the model. Structural elements account for "Knowledge Types", each of which representing a state that an evolving theory can assume. Functional elements account for the mechanisms required for each agent to contribute for the theory evolution; The whole formalizes what we have called a Reasoning Framework, the current abstraction for the notion of Contractual Framework introduced in $\S 1$;

- A perspective of the exchanges that can take place between the Artificial Agent and a Human Agent (referred as to the Learner) while a theory is built/revised as a consequence of contradiction detection and overcoming. Such a perspective is based upon the speech acts ask and tell, which role is to model messages that carry out the Knowledge Types mentioned above.

Considering the scientific rationale based upon the iterative and (eventually) converging cycle "experiment-and-theory", three main phases are proposed to support the exchanges among the agents: (i) Collecting information, (ii) Analyzing and Prototyping, and (iii) Testing and Revising. Let us consider these phases in the context of a dialogue between a learner Human Agent and his/her Artificial Agent, during which the former one drives a study about a certain concept.

\subsection{Collecting information}

In this phase, the information available to feed the theory formation process is collected. This might be or not a process allowed by the Artificial Agent. Let us consider the negative case, and, thus, we shall not consider the exchanges that could model the Collecting phase. 


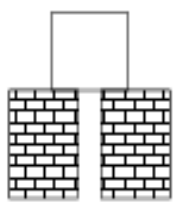

(a) Example 1

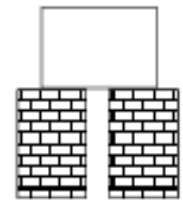

(b) Example 2

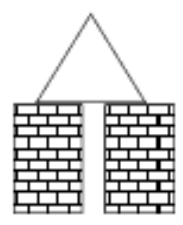

(c) Example 3

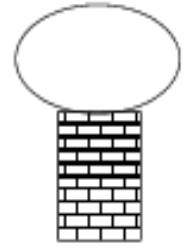

(d) Counter-example 1

\section{Figure 2: Hypothetical available objects to begin the study of the concept "Arch".}

Let us now start our hypothetical scenario by supposing that a History student interested in historical monuments intends to formalize the concept of "Arch". Let us suppose that, during a trip around the world, the student has taken some pictures in order to begin the study. The hypothetical images are those of Figure 2.

\subsection{Analyzing and Prototyping}

This phase should lead to (i) a Hierarchy of Terms representing the vocabulary supporting the study, and (ii) a Set of Constraints, which role is to constrain the usage of those Terms, as the constraints achieve formal relations among the Terms. The sub-phases leading to such structures are described below.

\subsubsection{Hierarchically organizing a vocabulary}

To go on with the hypothetical scenario introduced above, let us suppose that the student considers each object as being composed of a number of pieces, each one generally named, say, a form. Then he observes that the "forms" can be grouped together according to a classifying criterion, for instance, "to distinguish the forms that should roll when put on a planar surface from those that should not". By using such a criterion, the student could obtain on the one hand, stable forms (square, triangle, rectangle, block), and, on the other hand, unstable forms (oval). Such a student's reasoning would lead to a hierarchy as the one shown in the left-hand side of Figure 3. The exchanges needed for the agents to formalize such a hierarchy are shown in the right-hand side of Figure 3.

\subsubsection{Constraining a vocabulary}

The model suggests that, in a given moment, a theory is represented by an Axiomatics. Considering the dynamical character of a theory, Axioms may join or leave an Axiomatics, according to experiments carried out. The model allows for Axioms to join an existing.

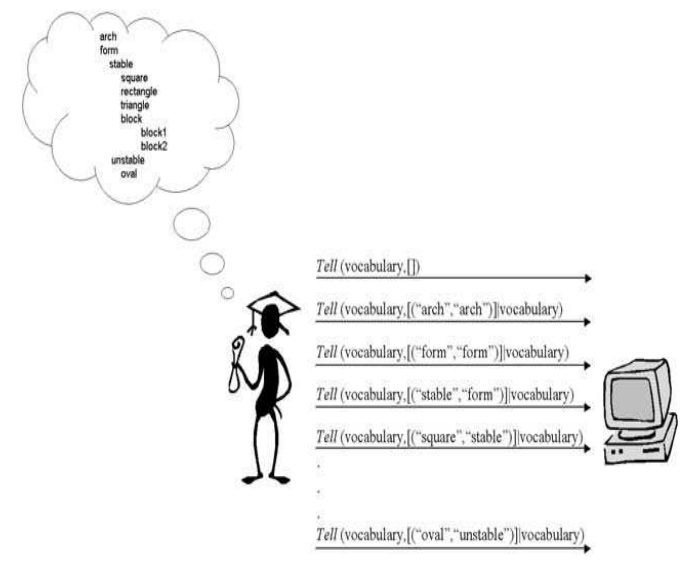

Figure 3: The Learner tells the Artificial Agent the vocabulary supporting the study.

Axiomatics, according to experiments arried out. The model allows for Axioms to join an existing Axiomatics either in a direct or an indirect anner. The former case accounts for the situation in which the user is able to identify a certain elation between the objects being studied, and then build up the corresponding constraints to stress the relations. The latter case accounts for the situation in which the user recalls the Artificial Agent's learning skill in order to look for suitable relations. Hereafter, we show the exchanges modeling the indirect case, to which the following subyphases take place: (i) Describing Examples to the Artificial Agent, (ii) Building up Constraints out of the Examples, and (iii) Filtering Learnt Knowledge.

\section{Describing Examples to the Artificial Agent.}

In order to show up the exchanges allowing the Human Agent to describe an object to the Artificial Agent as an Example, let us consider the object named Example1 from Figure 2(a). These exchanges are shown in Figure 4: Example1 is described by stating that a square is present, a first block is present, a second block is present, and an arch is present. The description of an object corresponds in the model to the Knowledge Type "Theorem", standing for a theorem to be proved out of the Axioms representing a theory. Once a Theorem is 
built, it may become an Example, such an operation modeling the fact that the Artificial Agent should memorize the object for latter use.

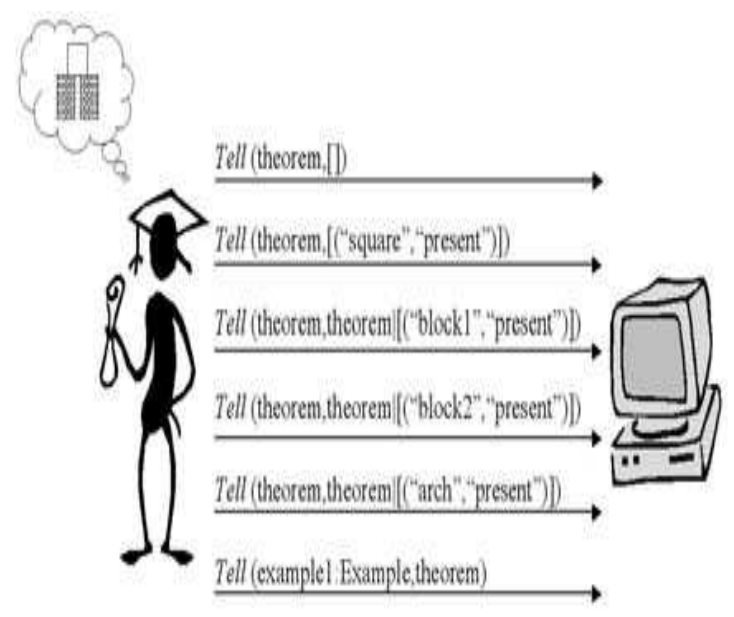

Figure 4: The Learner proposes an object to the Artificial Agent.

The Artificial Agent proposes a number of Constraints. Let us assume that the Artificial Agent knows the Examples representing the objects of Figure 2, and thus it is ready to learn general rules (Constraints) about them. The model Phi-calculus assigns each learnt rule to a Knowledge Type "Lemma". The exchanges between the agents are shown in Figure 5, over two illustrating Constraints that could have been learnt from the provided descriptions of the objects from Figure 2.

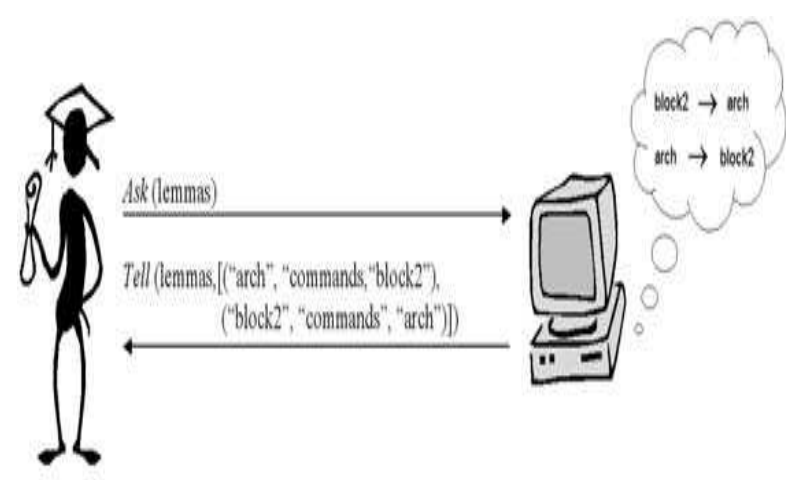

Figure 5: The Artificial Agent tells Lemmas to the Human Agent.

The Human Agent filters the Learnt Knowledge. Once he is informed about learnt Lemmas, the Human gent may analyze them in order to compose what is formalized by the Knowledge Type "Conjecture". A onjecture should retain only those Lemmas estimated by the Human Agent as pertinent. Once the analysis is over, the resuting Conjecture is memorized by the
Artificial Agent as an Axiomatics ready to be exploited. In Figure 6 we show the exchanges supporting the composition of a Conjecture and then its status changing to become an Axiomatics. In our scenario, we suppose that the Human Agent accepts as a Conjecture (then as an Axiomatics) both two Constraints proposed by the Artificial Agent.

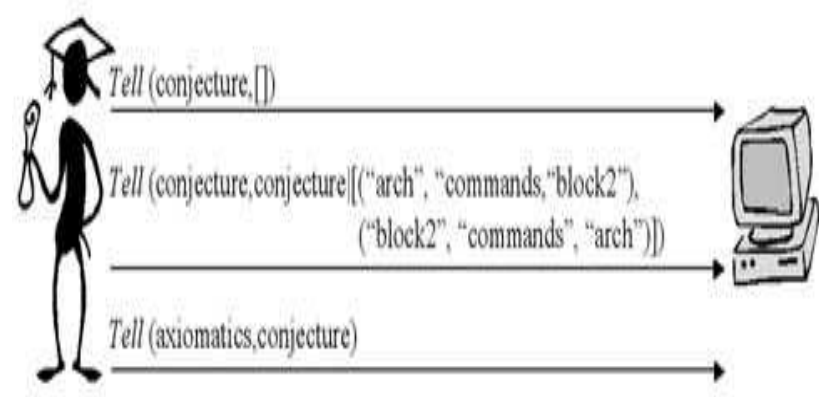

Figure 6: The Learner tells the Artificial Agent a Conjecture.

\subsection{Testing and Revising}

Up to this point we have shown Phi-ncalculus through some of the exchanges required to build a theory. As stated before, the model assumes that a theory is something constantly evolving as a consequence of experiments carried out. Once the Artificial Agent knows an Axiomatics, the Human Agent may then test its validity, by proposing a number of objects unknown by the first agent and then asking this agent about the object's Adequacy with respect to the current Axiomatics. The exchanges modeling such a situation are shown in Figure 7.

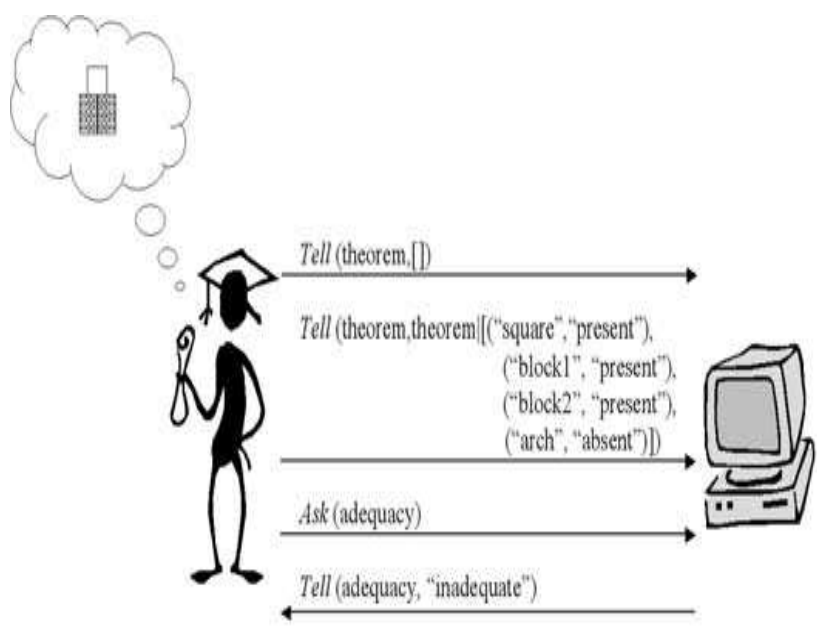

Figure 7: Learner proposes an unknown object to the Artificial Agent, who judges the object as Inadequate. 
The testing object, proposed through the Knowledge Type "Theorem", is the one shown on the left-hand side of the picture. The figure shows as well the exchanges allowing the Human Agent to know the object's adequacy (with respect to the Artificial Agent's current Axiomatics).

At this point we reach the heart of Phi-calculus as a contradictionydriven approach to theory formation. The Inadequacy of an object declared by the Artificial Agent lies on the basis of a contradiction revealed while the agent confronts the object description with the current Axiomatics. A revision process should then take place in order to reach a coherent behavior for the Artificial Agent. Such a revision process would require, however, the Human Agent to know how to reestablish the coherent status of the theory. As this is not always evident, before such a revision process could take place, the Human Agent may need to find out why a contradiction arises. The exchanges are shown in Figure 8, in which the Human Agent asks the Artificial Agent the reasons of its judgment.

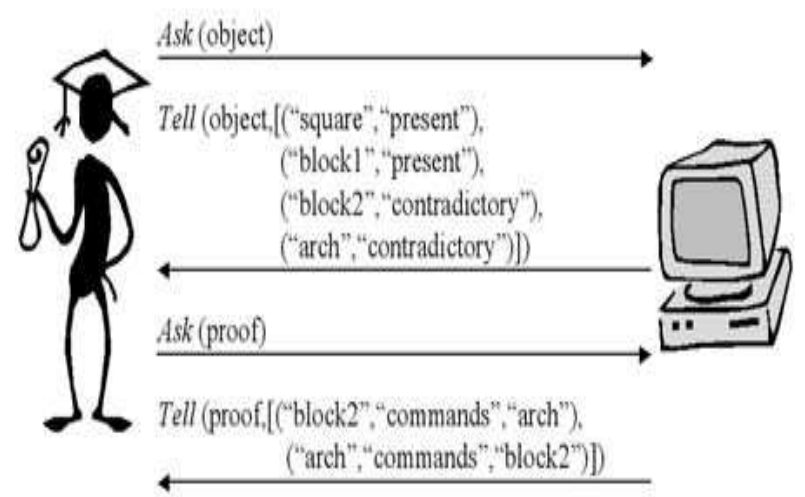

Figure 8: The Learner asks the Artificial Agent the reasons of its judgment; the Agent answers.

By means of the Knowledge Type "Object", the Artificial Agent shows how the proposed object looks like to it: the resulting description is a result of both the description from the Human Agent and the propagation of the Constraints (Axioms) from the current Axiomatics. In our scenario, the Term Arch is evaluated both as present (as a consequence of propagating the constraint Block2 $\rightarrow$ Arch) and absent, (from the Human Agent's description), thus, contradictory. Moreover, by means of the Knowledge Type "Proof", the Artificial Agent shows to the Learner the Axioms causing its judgment (i.e., the violated Constraints).

Having assumed the theory as over-constrained, the revision process consists for the Human Agent to tell the Artificial Agent to forget unsuitable Axioms. This may be a relatively simple way of revision. A more complex revision process is the one requiring to go back farest in the theory formation process, for instance, the need to reformulate the vocabulary and then to reformulate the Examples' descriptions, and yet to ask the Agent to renlearn over the Examples, and so on. In fact, this whole reformulation would be the case if we would go on with our scenario, since, provided that we would relax the Axioms responsible for Inadequacy, the Artificial Agent would not be able to decide about the property of being an Arch neither for Example 1 nor for Countery-Example 2. Excepting the evaluation of the Term Arch itself, the description of these two objects are quite similar, so that the learnt rules could not capture their distinctions.

\section{A higher-level abstraction perspective for Phi-calculus}

In the previous section we introduce Phi-calculus as a model for the design of DLEs under a particular perspective: the one of the exchanges taking place between a Human Agent and his/her Artificial Agent during theory formation process. By means of those exchanges, i.e., the messages sent/received by the Agents, the Knowledge Types composing the model are introduced, as well as the way they relate to each other. In this section we propose to isolate those Knowledge Types along with their relation to each other, in such a way to abstract the model from a scenario view. We believe this higher-level abstraction to be useful since it would allow one to envisage a variety of scenarios different from the one presented in $\$ 2$. Before concentrating in those Knowledge Types, however, let us briefly recall some notions from the history of sciences that have inspired some of our choices.

\subsection{Theorethical foundations}

To recall history is a very hard and dangerous task. When trying to do so, one takes the risk of naively omitting in his/her report people/work/events as important as those mentioned. That is the reason why we are definitely not the better placed ones to accomplish that task. On the other hand, however, since we borrow from that history a number of notions around which a great number of reflections have been taking place, we feel thus invited to take the risk and to highlight some of them.

Dialectics, Contradiction, and Concept. In $\$ 2$ the concept of Arch is exploited to introduce the model Phi-calculus. Alternatively, the concept of Bridge 
could have been used to motivate additional discussion about bringing up different concepts over a single vocabulary. In both cases, the notion of concept would be available to be handled by the user interacting with the system. However, it is also the case that the notion of concept have inspired the design of the model Phi-calculus itself. This should be rendered more explicit in what follows. Let us start by considering how philosophy has brought together the notion of concept with the one of contradiction and that of dialectics.

Around the notion of dialectics, two main phases can be distinguished from philosophical thought: Greek origin of the term and Hegelian tradition [10, 29, Dialectique]. The first one is concerned with the philosophy rationale: the dialogue drives a game in which each opponent tries to win by leading the other one to a contradiction. Platonian tradition put the dialectics as the art of learning to speak and to think, based upon the dialogue.

The second phase around the notion of dialectics begins with Hegel, who sees "false" as a necessary step for attaining "true". For Hegel, the thought is developed dialectically in a ternary rhythm: statement (thesis), negation (antithesis), and negation of negation (synthesis). Instead of a method, the dialectics is a process for producing "true", out of contradictions. For him, every contradiction is built-in knowledge and, since the concepts are at the heart of knowledge, concepts are articulating contradictory thoughts. Hegelian dialectics has then as its center the notion of contradiction in detriment of the notion of dialogue [10, 29, Dialectique].

With respect to philosophy of sciences the major interest on Hegelian dialectics is mainly due to the constructive role assigned to negation in concepts formation, as well as the status accorded to error as a moving element of knowledge [10, 29, Dialectique].

Considering work from which Computer Science, and particularly Artificial Intelligence, can benefit, the notion of contradiction has been considered for around forty years ago within a Logical context, including the so-called Paraconsistent Logics [11, 41]. With respect to those achievements, the main difference to what we are proposing is that the known formal systems are able to handle contradiction in reasoning, while Phi-calculus in its current state is not. Contradiction is instead placed as an indicator that some revision is needed in current available knowledge.

In his book Theories of concepts [45], Morris Weitz states that one can find a theory of concepts in the thought of every philosopher [29, Concept]. In fact, a number of philosophers have elaborated about the origin of abstract and general ideas: by abstraction, mind is able to isolate stable sets of characters shared by a number of individuals, and assign a name to each of these sets. Each set thus assigned by its name is a concept [10, Concept].

Kantian thought, for instance, distinguish between a priori experience-free concepts, and a posteriori or empirical concepts. The former ones refer to a priori conditions of every knowledge, e.g., plurality, causality, etc. The latter ones, coming from experience, account for classes of objects or beings, and that is the reason why we often analyze them in terms of comprehension and extension [10, Concept].

In contemporary epistemology, concepts are often considered under a theory of definition. A major question in contemporary sciences, relating the notions of concept and that of law, is that certain concepts seem to be conceived under the form of definitions involving conditional statements, as discussed by Hempel in his article "Fundamentals of concept formation in empirical science" [20]. Such a relation between concept and law has yet been highlighted by Sellars [39], who states that concepts always imply laws, without which they would be empty and undistinguishable [29, Concept].

Nowadays, the term concept is often used to refer to designing results, to the extent considered in engineering. Such a current tendency seems to expand the usage of the notion beyond scientific contexts, like those of techniques and technologies [29, Concept].

Recalling contemporary sciences, particularly cognitive sciences, efforts are yet invested in order to understand how human acquire and represent knowledge (c.f. [28, Chapter 1]). Under a symbolist view, human knowledge is organized in hierarchies of concepts or categories. In such a context, a consensus seems to turn around the notion of categorization: to categorize is to group individuals aiming a certain objective, particularly in order to facilitate reasoning out of the established categories. Following that thought, a category refer to a number of individuals sharing several features. These latter are achieving a necessary and sufficient condition for an individual to be included into a category: its definition. Such a definition of definition of a category seems to correspond to Kant's comprehension axis for concept's analysis.

In our work, we retain thus the idea of "definition in comprehension" and "definition in extension" of a concept or a category to refer to, respectively, (i) the set of rules necessary and sufficient to determine the inclusion/exclusion of an individual and (ii) the set of individuals such that corresponding descriptions respect the rules. We retain as well the idea of definition (in comprehension) of a category or a concept in terms of laws, following the claims from contemporary epistemology. 
There exist, however, an additional view of categories, which supposes the existence of a similarity measure between individuals, preceding the categories themselves (c.f. [28, Chapter 1]). This view is on the basis of the theory of prototypes. A prototype is an individual in the average the more similar one to each other individual in the category. Such an individual is said to be the most representative one of the category. According to this view, a category is given by a prototype and a floor. All the individuals close enough to the prototype (those having a similarity measure greater than the floor) belong to the category. We retain as well the definition of a category or a concept in accordance with that vision.

Two approaches from scientific rationale. In this section we recall the two main approaches from scientific rationale grounding the dynamics underlying Phi-calculus, namely, (i) empirical sciences rationale (as initially elaborated by the empirist movement) and (ii) Popperian hypotheticaldeductive approach.

As well-known, the so-called contemporary sciences' method has its origins at the beginning of XV IIth century, with the ideas from Bacon [9]. According to Bacon, one would reach knowledge by collecting facts, by organized observation in order to elaborate theories. The movement known as Empirism is consolidated in the following century, claiming the exclusion from knowledge of anything not reducible to experience. Such a movement has initially lied on the verifiability principle, replaced later by that of confirmability. [10, Cercle de Vienne, Carnap Rudolph].

In [36] Karl Popper elaborates a criterion allowing one to consider a theory as a scientific one. Popper's "falsiability criterion", also known as the "Refutability criterion", becomes the heart of a philosophical movement which is opposite to Empirism: the scientific rationale is not inductive at all - it does not proceed by means of repeated observations and law formation. It is, on the contrary, hypotheticaldeductive: stating audacious conjectures comes at first, being such conjectures submitted then to experiments. While it had not been refuted, a scientific theory is nothing but a corroborated hypothesis [29, Popper Karl Raimund].

While Popper had influenced knowledge in sciences with his Refutability principle as a criterion for deciding about the scientific status of knowledge, Imre Lakatos had contributed to knowledge in Mathematics. Lakatos had elaborated a logic of discovery in Mathematics also based upon the Refutability principle. His "Proofs and Refutations" logic [23] considers that knowledge in informal Mathematics is hypothetical, conjectural, and is developed by means of speculations and criticisms.
The objective of such a logic is to study the construction of a proof and the establishment of its validity domain, through the analysis of examples and counter-examples to the proof. In this context, a proof is seen as a set of lemmas established by analyzing a conjecture and its sub-conjectures. The analysis of a proof is accomplished by proposing examples and counter-examples, in such a way to discover and to modify lemmas, according to the criticisms or the refutations to which the proof is submitted.

As one could notice, the "Proof and Refutation""s method demands an iterative and interactive approach, particularly, a dialectic approach, since it is based upon, on the one hand, on the elaboration of a Conjecture's Proof and, on the other hand, on the criticisms for such a Proof.

\subsection{Knowledge types and their relations in Phi- calculus}

In this section we introduce an elaboration for the model Phi-calculus under a more abstract view than the one presented in $\$ 2$. As stated above, the aim of such an elaboration is to allow one to think of the model in a kind of scenario-free perspective, such as to be able to envisage other exploitations in terms of scenarios and potential applications different from the ones presented in the paper. Such a perspective for Phi-calculus (Figure 9) is grounded on the theoretical foundations from $\S 3.1$.

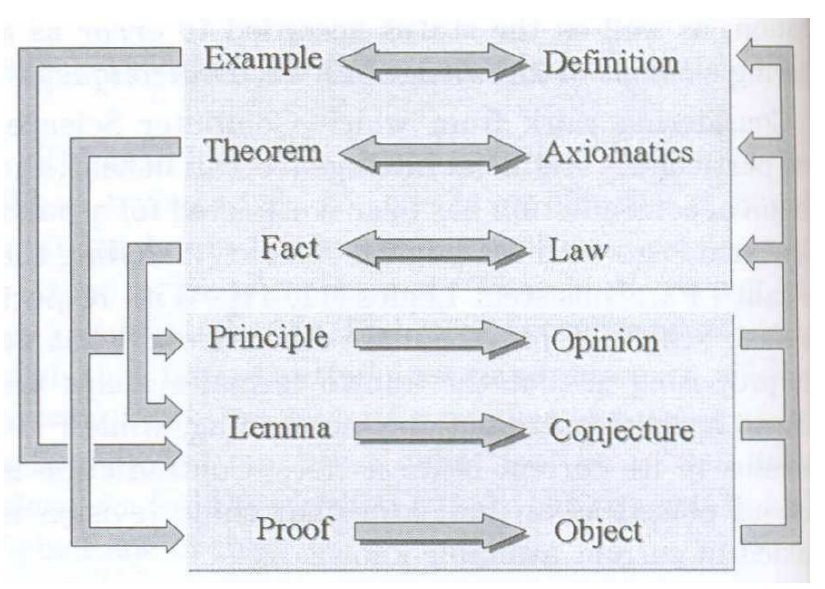

\section{Figure 9: Knowledge Types and their relations in Phi-calculus.}

Considering that each Knowledge Type represented in Phi-calculus gains its sense only when thought-of within the relations it keeps with the others, the heart of the model lies on such a theory formation "ontology". As a matter of choice, we privilege in the following narrative the dynamics underlying the model than its structural features. Therefore, rather than concentrating in each 
Knowledge Type, one might observe the overall idea of how the two scientific rationale discussed above are brought together in order to compose the model.

One possible way of analyzing the model is to consider a horizontal cut that would separate the Knowledge Types into two groups of six Types each. The upper segment (see Figure 9 ) accounts for those Types and relations involved in the Theory Testing process. This segment might be, by its turn, vertically divided into two sub-groups: Example, Theorem, and Fact (the Testing part), and Definition, Axiomatics, and Law (the Theory part). The Adequacy criterion relating experiment to theory $(\$ 2)$ is represented by the horizontal arrows relating one Type from Testing part to another one from Theory part.

The lower segment from Figure 9 is representing the Theory Revision process. Similarly to the upper segment, one might divide it vertically into two subgroups as well: Principle, Lemma, and Proof, and Opinion, Conjecture, and Object. As one might also notice, the Knowledge Types represented in the lefthand side vertical sub-group are obtained from the corresponding vertical sub-group in the upper segment (such a generation is represented by the topdown arrows). This is to capture the idea that the elements often used to test a theory may be conveniently used to feed a revision process. As a result of analysis, revised knowledge is generated (generation is represented by the lower horizontal arrows) and yet additional analysis should take place in order to decide how to accommodate such revised knowledge within existent knowledge (accommodation process is represented by the bottom-up arrows).

In order to reinforce the above mentioned suggestion, we believe that the way of typing knowledge and relating these types is really "just" a matter of choice. Of course, such a "just" should involve considerable investigation aiming at appropriate grounding, which is suitable since it would minimize the risk of eventual unsuccessful instantiations of the model. We think that the major importance within the proposed model is the way of bringing together in a synergetic manner two classical approaches on how scientific knowledge evolves. One possibility for further investigations would be to find out the limits of such a theory formation "ontology" by submitting it to application fields, as we started to accomplish in human learning.

Potential scenarios. In the beginning of this section we argue on the interest of designing Phicalculus under a scenario-free perspective on the basis that such a view would allow one to envisage a variety of scenarios over the model. In what follows we reinforce this argument by presenting some of the scenarios that had been thought-of considering the domain of Human Learning. These are illustrated in Figure 10 .

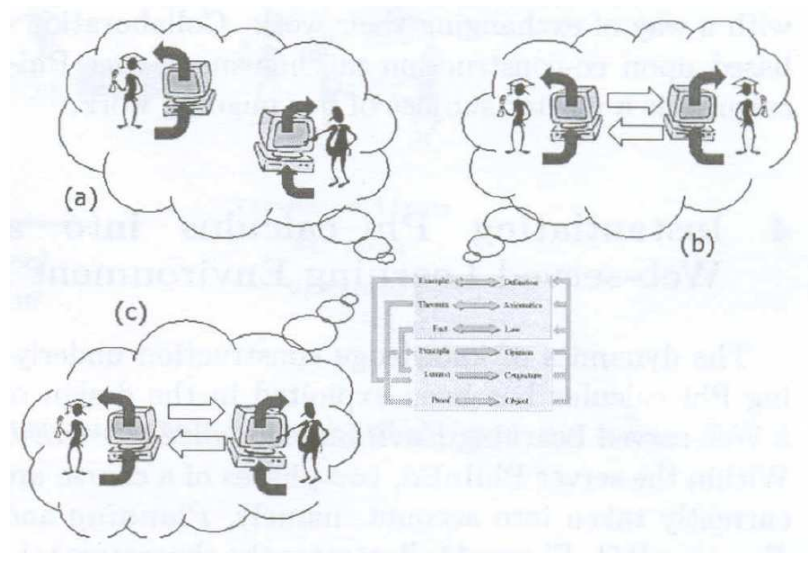

Figure 10: Some possible scenarios over the model Phi-calculus within the domain of Human Learning.

The scenarios are depicted essentially in terms of the role played by the individuals taking place, along with the interactions between them. As we show in $\S 2$, these interactions would be designed on the basis of messages that would carry the knowledge typed according to the model. Scenario (a) involves both the Learner and the Teacher each of them interacting with an Artificial Agent (notice that the interactions between the Learner and his/her Artificial Agent correspond to those developed in \$2 ).Scenario (b) involves the Learner interacting both with an Artificial Agent and with a peer (through their Artificial Agents). The characters in scenario (c) are the Learner and the Teacher each of them interacting with his/her Artificial Agent and with each other (through their Artificial Agents).

In the following section we introduce a particular instantiation of the model Phi-calculus within Human Learning, more precisely, into a Web-served Learning Environment. This is achieved by combining both three scenarios which are organized according to the phases of a course.

At this point, it is important to notice that interactions between the Learner and his/her peer often suggest a collaborative context, as it is considered by the Educational Community. One possibility of exploiting cooperation within Phicalculus is highlighted within the above scenario (b). In order to actually account for this scenario on the basis of the model, a co-construction mechanism should take place, in addition to an individual construction like the one presented in $\$ 2$. The current implementation of the server presented below allows "collaboration" only in a more strict view: students are provided with ordinary communicating 
mechanisms (like electronic mail), and also with a way of exchanging their work. Collaboration - based upon co-construction mechanisms - over Phi-calculus is a matter subject of our ongoing work.

\section{Instantiating Phi-calculus into a Web-served Learning}

The dynamics of knowledge construction underlying Phi-calculus has been exploited in the design of a Web-served Learning Environment called PhiInEd. Within the server PhiInEd, two phases of a course are currently taken into account, namely, Planning and Running [15]. Figure 11 illustrates the characters taking part in these phases, along with the actions performed and the possible relations to each other.

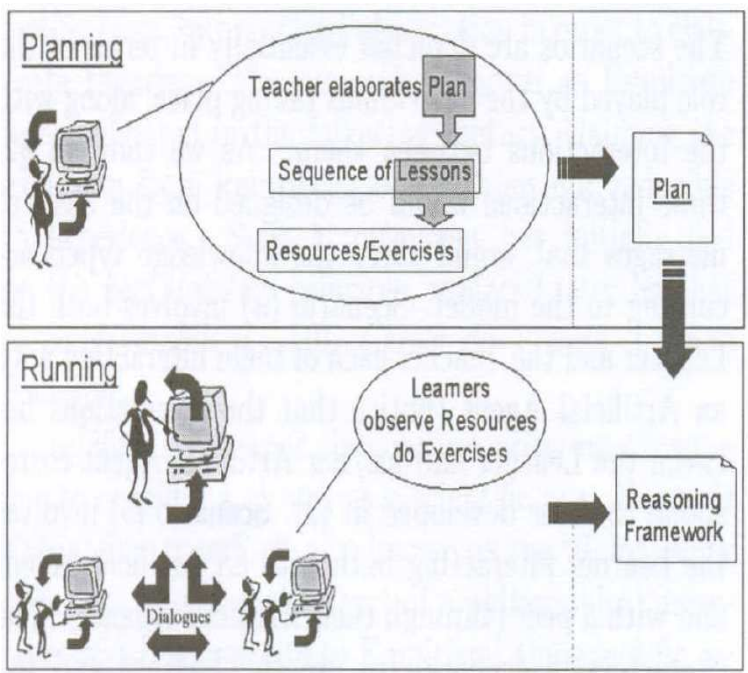

Figure 11: Two phases of a course supported by the server PhiInEd.

\subsection{Planning}

Planning a course within PhilnEd consists of the elaboration of a Plan by the one who administrates the course, to which we refer as the Teacher. A Plan consists of a sequence of Lessons, to be studied by the ones who follow the course, each referred to as the Learner. The Teacher may provide each Lesson with a number of Resources and/or Exercises. Both Resources and Exercises are concretely provided by the server through Web pages. Resources may be seen as the course's content, while through Exercises the Teacher asks the Learners to react with respect to Resources. Learner's reactions are memorized by the server for later user by the Teacher/Learner's peers, corresponding to what we call a Reasoning Framework in $\S 1$.

Altogether - observation of Resources and construction of a Reasoning Framework - are expected to stimulate the development of certain capabilities of the Learner. The enumeration of these capabilities constitutes the list of the Objectives of the course. Then, the Lessons established should be those estimated by the Teacher as capable of leading a Learner to reach the Objectives of the course. When elaborating the Plan, one possibility for the Teacher could be to think firstly in terms of Global Objectives, and then to decompose it into a list of Local Objectives from which the Lessons of the course would be elaborated.

\subsection{Running}

Running a course within PhilnEd consists of the execution of the course's Plan, causing the Learners to study its Lessons, guided by the Teacher. Along the Running phase, individual work is provided by PhiInEd such as to enable the Learner to elaborate a Reasoning Framework as a consequence of observing Resources. Communication among participants is provided such as to allow (at least) both the Teacher to propose a Lesson to the Learner, and the Learner to present his/her RF to the Teacher and/or to his/her peers.

The social level. The Running phase of a course is organized as a Sequence of Dialogues. Subjects of Dialogues correspond to the title of the Lessons from the Plan. For instance, a course which Plan has three Lessons, will be executed within three Dialogues, one for each Lesson. A Dialogue is composed of a sequence of Messages, and the Subject of a Message is, like for the Dialogue containing it, the title of a Lesson. The Teacher may declare a Lesson as Studied by a Learner, when the Dialogue about that Lesson has at least the two following Messages: (i) from the Teacher to the Learner, transporting the Lesson to be studied, and (ii) from the Learner to the Teacher, transporting the Reasoning Framework of the Learner corresponding to the Lesson subject of the Dialogue. Besides these two Messages, additional ones may occur in a Dialogue, in cases where a discussion takes place between the Teacher and the Learner about the study. 


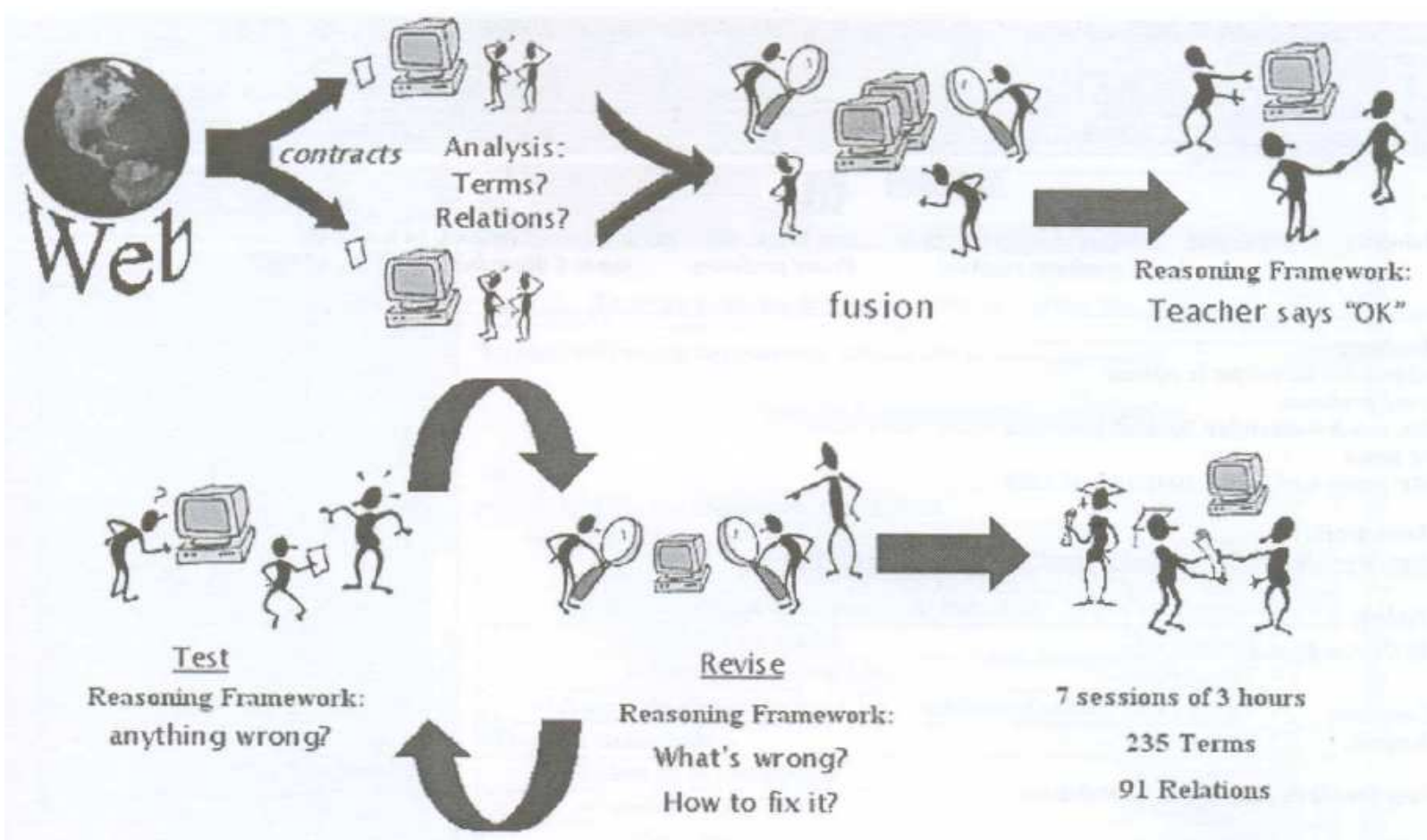

Figure 12: Studying scenario about the contractual techniques over the server PhiInEd, involving a Law D.E.A. class.

Remark. Let us highlight that while the Teacher uses PhiInEd to elaborate (during the Planning phase) a Reasoning Framework particularly called a Plan, the Learner uses the same tool from the server to elaborate a Reasoning Framework (during the Running phase) as a result of studying a Lesson. The distinctions between the two Reasoning Frameworks rely on the vocabulary and on all elaboration coming as a consequence. While the vocabulary related to a course's Plan includes terms like Lesson, Exercise, Task, Objective (and eventually content terms), and so on, the vocabulary handled by the Learner would be the one related to the content he/she is dealing with while studying the Lesson.

\section{A contradiction-driven study of Contractual Techniques in Law}

In this section we focus on the interest of Phiycalculus for the design of Discovery Learning Environments by reporting a real study situation held upon the server PhiInEd. We leave the hypothetical scenario developed in $\S 2$ upon the block's world, and we recall the Law domain, already evoked in $\$ 1$. Here, a pedagogical context is voluntarily created. A class of twenty-seven students of D.E.A. (Diplôme
d'Etudes Avancés), from Université Montpellier I, Montpellier (France), under the supervision of Prof. Dr. Didier Ferrier (referred as to the Teacher), have been using the server during seven sessions of three hours.

The subject of study. The chosen subject of study for the course was the so-called General Conditions of Sale (GCS). The definition of GCS requires the understanding of the process of formation of a contract. This latter is established whenever an offer meets an acceptance resulting in an agreement that constrains the behavior that the two sides intend to adopt with respect to the other. In such a context, the GCS are defined as an offer of contracting addressed by a seller to any buyer interested on acquiring his products. This offer constitutes then the individual norm of the behavior that the seller intends to impose to his potential buyers. The unconditional adhesion of the seller's conditions by a buyer should be enough to form the contract, and the individual norm composed of the seller's conditions becomes then the norm common to the two parties.

The scenario. (Figure 12 ) The Teacher has initially chosen the GCS as the subject of study. A Reasoning Framework (RF) should then be constructed for this contract class. The goal was to obtain a single RF as a result of the work of the whole group. Firstly, students were distributed in seven working groups, each group working around a 
single machine. Each group should then prepare a RF by analyzing a GCS document from the Web. Secondly, a single RF should be generated as a fusion of RFs from the groups. Finally, this resulting RF should be revised under the supervision of the Teacher, up to be considered by the group as stable.
The reader is invited to remark in the above scenario the similarity voluntarily kept with Fidal's method scenario (Figure 1 ). In spite of the fact that we are accounting here to a pedagogical context, a

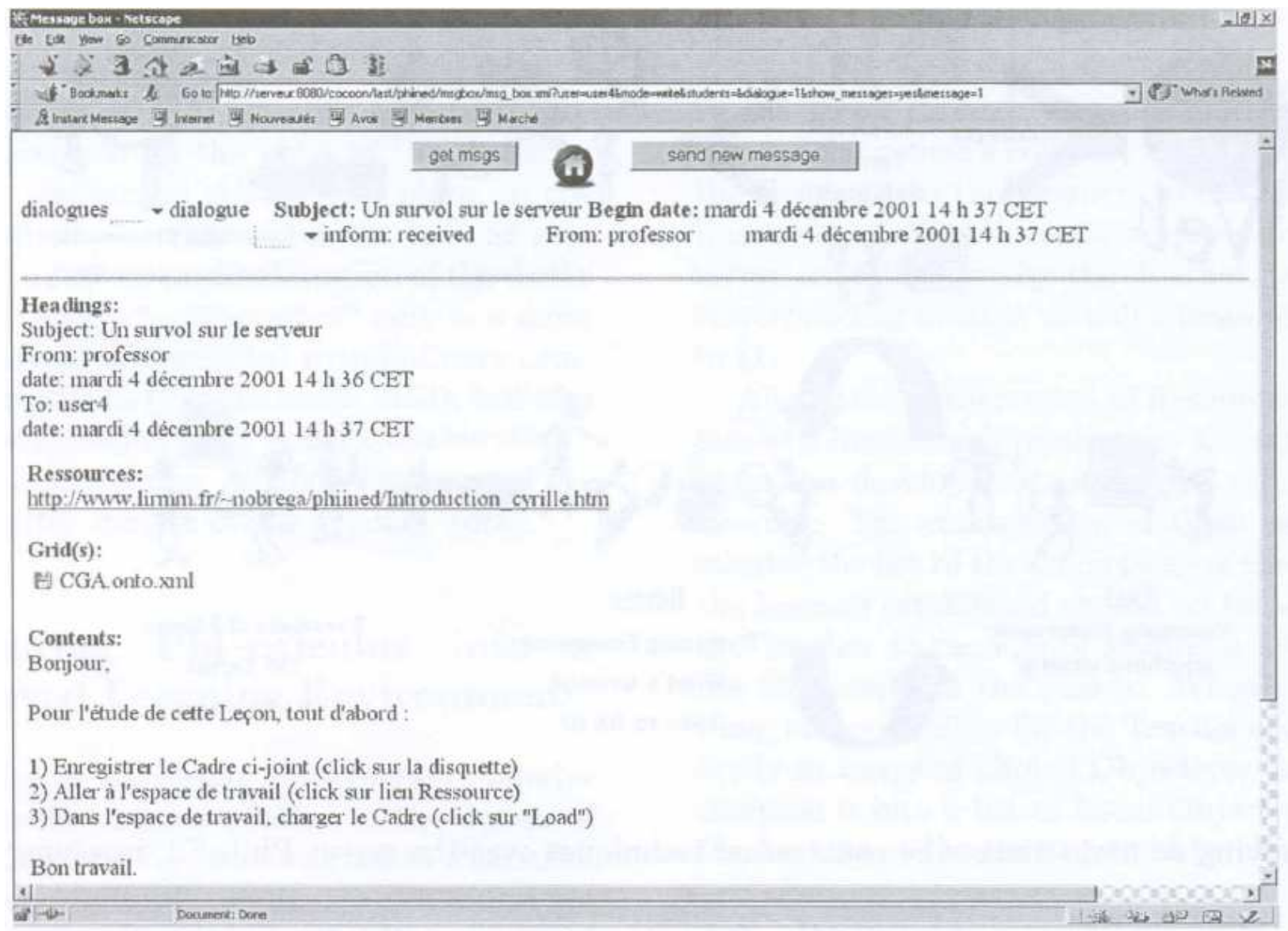

Figure 13: Learner's Message box: Message asking him/her to begin the study of Lesson 1.

significative difference lies on the fact that the source of Examples here is the Web.

Below, we describe the study carried out upon the server according to the two phases of a course supported by PhiInEd (as presented in $\S 4$ ).

\subsection{Planning}

Global Objectives. These were stated as "To be able to elaborate, criticize and improve particular contracts; to be able to apply general contractual techniques to specific contract classes." The resulting Plan. The course was planned along six Lessons, each of which is introduced hereafter, through its local objectives, Resources/Exercises, and a report on how the students carried it out. Notice that Resources are, in the server, Web pages giving technical support to the corresponding conceptual notion of Example from the model. Examples here are not Arch pictures, but legal contracts.

On the other hand, Exercises have no corresponding conceptual notion in the model, they are here as a way to provide some guidance for the students during the study.

\subsection{Running}

Lesson 1: An overview of the server. The Local Objective is to apprehend the server through its components, their functionalities and information they handle. By means of an example $y$ a very 
simplified RF of General Conditions of Buying $y$ all the components are covered for the students to become familiarized with the work needed to construct a Reasoning Framework. A Resource is supplied with an explanative text, which is available in a Web page, while the students explore the server through the RFy-example. Figure 13 shows a screenshot of the Learner's Message box: the Message asking the group to study Lesson 1. Students exhibited curious to discover PhiInEd, and some of them had preferred to work individually, instead of in groups. Also, they found it not evident to apprehend in a first moment "all the concepts" of the server.
Lesson 2: Let's start to work. The Local Objective is to stimulate the capacity of abstraction by analyzing examples. Concretely, to apprehend the notion of a good GCS from GCS documents. No Resource is foreseen for this Lesson. Part of a RF of General Conditions of Sale (GCS) is supplied, as a starting point for the work. Such part consists on a Hierarchy of Terms, voluntarily prepared to be incomplete. As an Exercise, the students are asked firstly to observe the Terms and how they are organized. Then to search on the Web, and then to retrieve, a page containing a GCS. They are instructed to analyze this page, and to improve the Hierarchy accordingly. Figure 14 illustrates the work from one group.

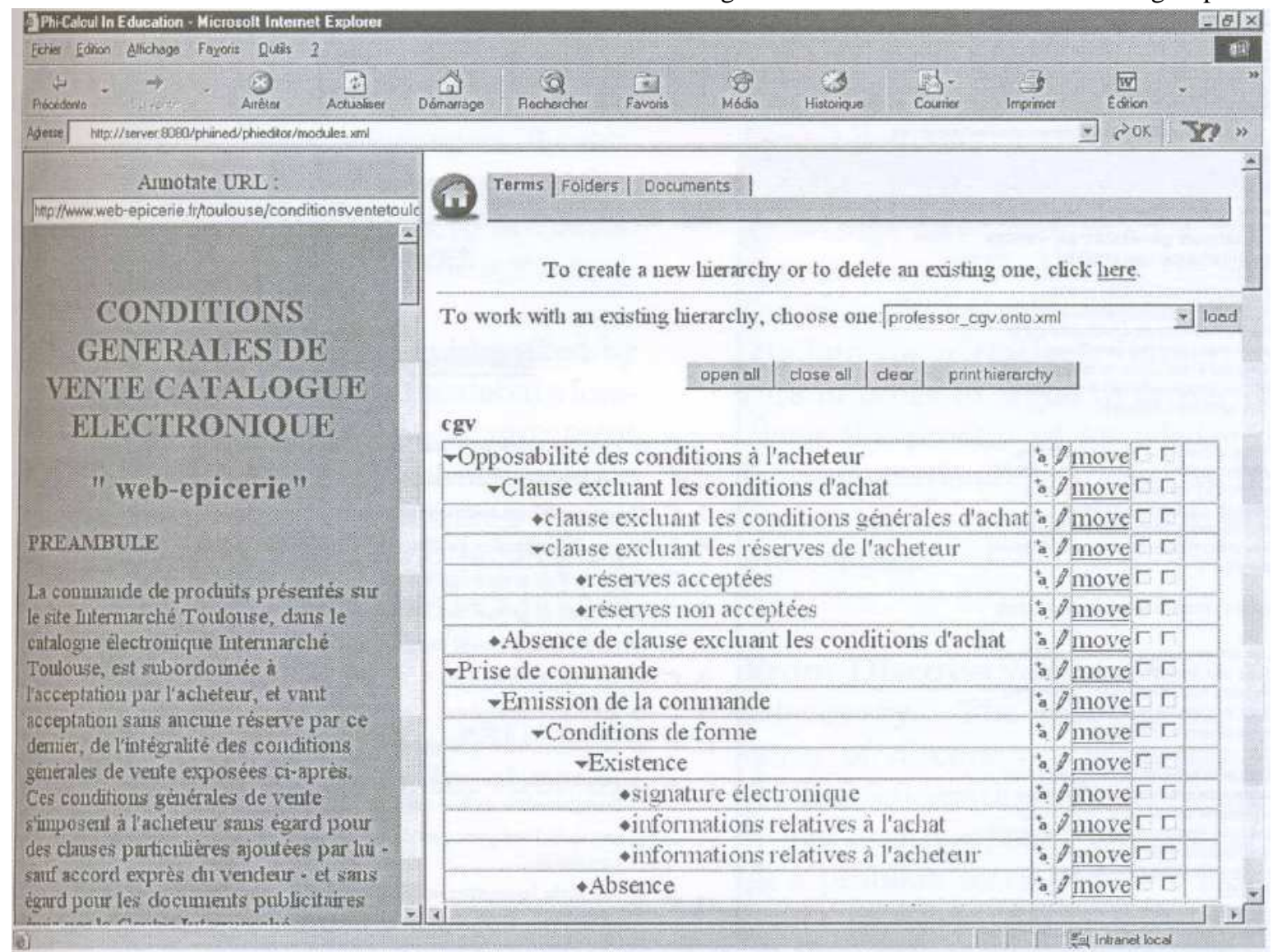

Figure 14: The work from one group: GSC document chosen from the Web (left-hand side) and Terms Hierarchy after some modifications (right-hand side).

Faced with a real case to work out, students found positive the fact of working in small groups, since they could discuss with each other. In some cases, discussions were even too long: 20 minutes before they could agree on adding a single Term! They became more familiarized with the server, and even suggested improvements concerning the edition of the Terms Hierarchy. This necessity was due to the dynamism on updating the initial Terms Hierarchy, in part caused by discussions, in part caused by the progressive analysis of the Web page they have chosen to study.
Lesson 3: Entering a Document, and Constraints. One Local Objective is to be introduced to the activity of exemplification, i.e.: to instantiate a contract in a RF. Another Local Objective is to learn the link between clauses in a GCS, by constraining the use of Terms in clauses, through the identification of logical relations among Terms. The single Exercise asks students to describe the contents of their working GCS document, by using the Terms of their working Hierarchy. The Exercise asks them as well to identify relations among Terms and then to build up the corresponding Constraints. Figure 15 
shows some of the Constraints from one group, who worked out a GCS document for spectacle tickets. An example of Constraint is "group fee excludes individual fee". Students exhibited an initial difficulty to build up Constraints, although these were introduced in Lesson 1 through a simplified RF. Such a difficulty was quickly overcame, due both to the practice, and overall, to the understanding that Constraints were nothing but a formal way of stressing relations among Terms representing clauses, something to which they are, as lawyers, actually familiarized.

Lesson 4: We present our results. A first Local Objective of this Lesson is to stimulate the capacity of comparison between an abstraction and an example. Concretely, since students have built a RF, they are supposed to have in mind the notion of a good GCS (at least their own!), and thus, they should be able to identify in GCS documents both positive and negative points with respect to their RF. Up to this point students work organized in seven groups. In this Lesson they are invited to work as a whole group. The single Exercise asks each group to present their RF to the larger group, to present their analysis about the RF they received, the Terms and Constraints they created, and yet to criticize the GCS they have chosen with respect to their own RF. Another Local Objective is to stimulate the capacity of argumentation and explanation, by means of debate: in case of different viewpoints (between groups) when students compare their RF with other's GCS documents, or even their RFs to each other. Before starting the expositions, the groups exchange their RFs by sending a message to each group. The results presented were more complementary than conflicting to each other. An interesting case was the one of two groups that had chosen, as a coincidence, the same Web page to work out. They had, as expected, some common results, but also they had perceived different aspects in the document, over which they finally agreed as complementary.

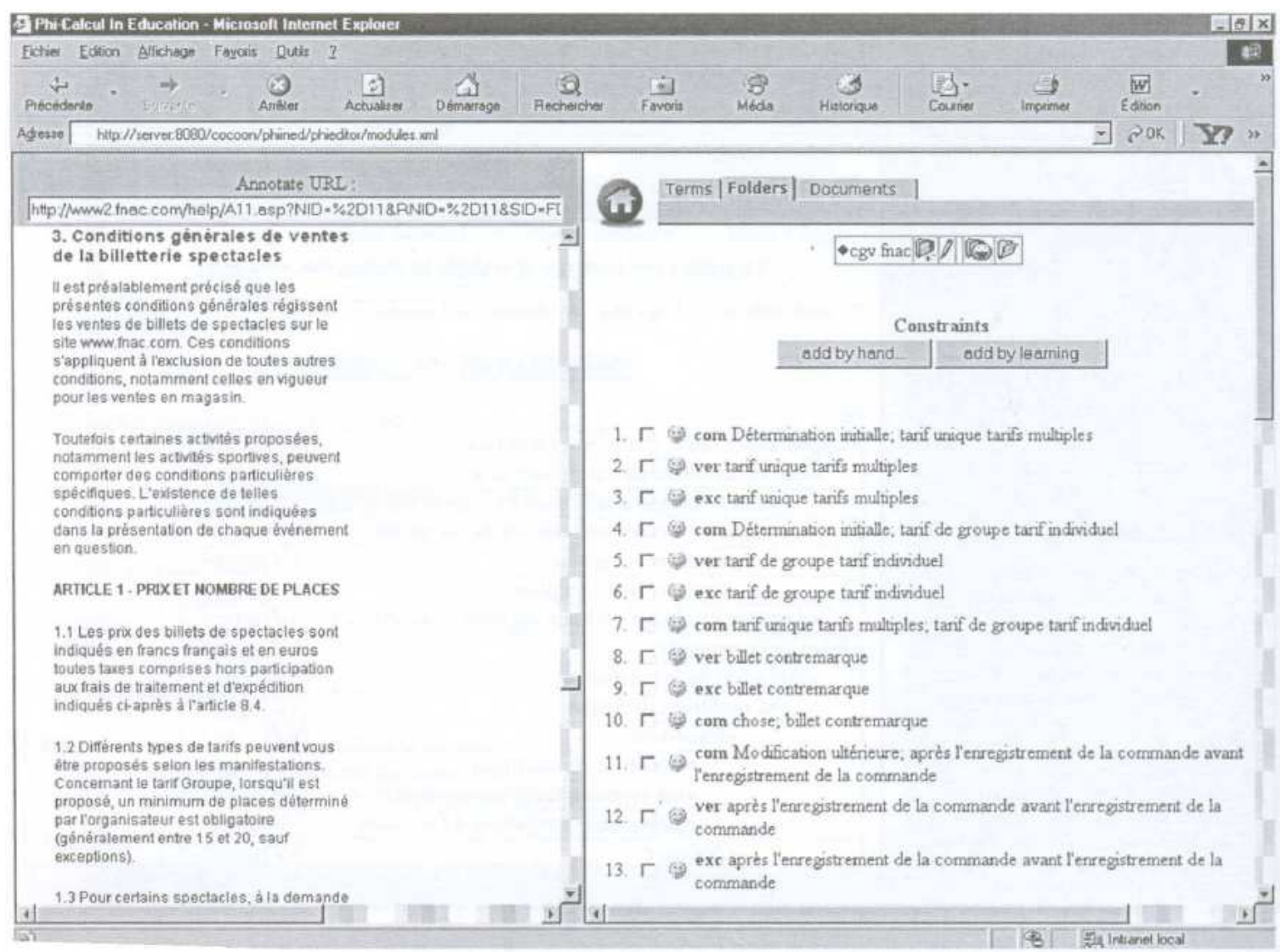

Figure 15: The work from one group: Chosen Web page of GCS (left-hand side) and some added Constraints (right-hand side).

Lesson 5: How about putting altogether? The Local Objective is to reinforce the capabilities stimulated in Lessons 2, 3, and 4: after knowing the others' work, students should formalize what they eventually apprehend from the debate with the larger group. Concretely, they should compose a reduced 
group responsible for generating a single $\mathrm{RF}$, as a result of merging the RFs from the groups. The single Exercise asks students to consider the RF of their corresponding group, and to create a new RF resulting from all RFs together.

Voluntarily, a member of each group presented himself to compose the reduced group. They have adopted the strategy of performing partial fusions (two by two) due to the amount of information they obtained as a result of a single fusion. Finally, the complementarity observed in the previous session was not so confirmed, since they discussed yet a lot, before arriving to a final result. This lead us to think that even if they do not agree with other's work, students hesitate in criticizing. The fact of working together around a common and concrete objective seems to provide an actual collaborative environment, in such a way to make them naturally criticize without having the feeling of "hurting" their peers

Lesson 6: Finally, did we reach an agreement? The Local Objective is to improve the notion of a good GCS that students have built up to this point. By proposing a number of GCS documents specially chosen to stimulate revision, the Teacher together with a group of several invited lawyers attempt to invalidate the Reasoning Framework representing the agreement among twenty-seven students.

Some specific points have hardly been identified by the invited lawyers in order to provoke the debate foreseen for this last Lesson, suggesting that an agreement was reached (achieving such an agreement represents is the model the criterion of a stable Reasoning Framework). Some improvements were orally pointed out but not performed on the RF, due to the lack of time.

\section{The contradiction-driven rationale beyond Human Learning: further investigations}

At a conceptual level, the work reported in this paper turns around the interactive knowledge construction, or yet, knowledge construction by means of human/computer collaborative work. Particularly, we approach knowledge construction through a theory formation perspective, which is grounded on the inductivist rationale brought together with a hypothetical-deductive approach. Additionally, we believe that the intended synergy for such a compound comes from the notion of contradiction, as it is on the basis of knowledge evolution allowing the dynamics of the overall process. As a result of our conceptual work, a knowledge construction model Phi-calculus - is proposed, relying on knowledge evolution along human/computer interaction being driven by contradiction detection and overcoming.

In previous sections, we show how Phi-calculus may be instantiated within a particular domain, the one of Human Learning. For such, we proceed as follows: (i) firstly we observe a well-succeeded practice (Fidal's method) aiming at examining it with respect to the discipline's state-of-the-art; (ii) then we actually instantiate the model by means of a scenario view that motivates the design and implementation of a Web-served Learning Environment and (iii) as a third step, we submit such a tool to a real case of study in Law.

In the current section, we point out to the potential of the model of being exploited in other domains, namely, for the design of Systems for aiding Discovery, within the field known as Computational Scientific Discovery (CSD). The reason we keep the discussion under a perspective of the model's potentiality considering CSD, is that the third above step carried out for Human Learning is yet to be accomplished for that former domain.

Although the current discussion is about scientific knowledge and its emergence, we invest less effort on approaching CSD through the scientific rationale underlying Phi-calculus (although that appears implicitly into the narrative). Rather, we inspect the literature in order to argue in favour of user's intervention along the process of knowledge discovery supported by computational artefacts: we believe such an intervention to be crucial for contradiction detection and overcoming.

From Discovery Systems to Systems for aiding Discovery. The research on computational modelling of discovery process has strongly contributed for the automation of such a process. Decades ago, it has been proposed to consider a discovery system as a problem solving system [35] guided by heuristic search methods. AM [31], EURISKO [32], BACON [26], GLAUBER [27], and BOOLE [30] constitute some of the classical discovery systems largely known by the satisfactory results they exhibit on simulating historical discoveries from science. On the other hand, as stated by Simon, Valdés-Pérez and Sleeman [40], efforts are today needed to guide the development of systems capable of solving problems in cooperation with domain experts. According to the authors, such systems would play an important role in discovery processes since, when dealing with complex domains, there is no much hope to successfully embody into the system an achieved theory (even because it is constantly evolving). As a consequence, a system which design has accorded minor attention to the interaction with the system's user (or user's group) will possibly not succeed in accounting to knowledge evolution in its working domain. Thus, instead of designing discovery systems, an alternative perspective is to think of Systems for aiding Discovery, if one is interested on the system's capability to account for continuous evolution of its user's domain knowledge. In our teams, such an approach has been largely exploited to the design of systems (e.g. [19, 37, 12]) as Rational Agents [38], to the extent that they are able to build theories under the supervision of an expert. Indeed, theories that Rational Agents are able to build in cooperation with an expert, exhibit properties required by a scientific theory, namely predictability and explicability in the context of experimental improvement.

Jong and Rip [22] discuss the "computer-aided discovery environments" as a future designing 
perspective for integrating a variety of tools available for (a group of) scientists. As largely known, the scientific rationale includes a number of phases/processes: scientists identify a problem, then they must find an adequate representation for it, they collect data by observation or experiment, and then they find out regularities and generalisations describing data. As a consequence of the development of increasingly powerful AI tools, new possibilities are foreseen for discovery environments to support such phases/processes. For Jong and Rip, the set of integrated computational tools compose thus a computer-aided discovery environment. The authors highlight, however, the fact that such an integration should account for a "socio-technical system" view. The expression is borrowed from organisation theory [42] and, within the context of computer-aided discovery environments, it would refer to the way by which the available tools are brought together in order to be effective in practice. Jong and Rip propose a number of guidelines to the development of future computer-aided discovery environments.

Yet relevant recent work from the CSD community point out to the benefits of the computer-aided discovery approach. Such benefits appear concretely through the results obtained by a number of systems capable of supporting the accomplishment of totally original discoveries. For instance, Pat Langley [24, 25] recalls AI research into CSD and its recent application to the discovery of new scientific knowledge. As an evidence of the advantages of such human/machine cooperation, Langley reports seven examples of new (computer-aided) discoveries that have appeared in the corresponding scientific literature. He highlights the role played by humans in each case. Going further, Langley suggests five phases for computational scientific discovery in which human intervention may influence system's behaviour. The author explicitly recommends the computer-aided discovery approach, instead of criticizing human intervention, as often done in past AI.

In the same direction, Raúl Valdés-Pérez [43], analyses a number of discovery systems that had accomplished totally new discoveries by playing the role of collaborators of the scientists involved. By such an analysis, the author aims at extracting regularities that would serve to guide the design of future systems. Valdés-Pérez proposes thus a list of recommendations that, according to him, are there to complement those proposed by Jong and Rip in [22]. The guidelines are identified, yet according to the author, by proceeding his analysis under a perspective of individual collaboration scientist/program.

Discussion. From what is presented in this section, we highlight some basic premises as promising candidates to ground the work around interactive knowledge construction: (i) within the discipline known as Computational Scientific Discovery, the systems designed have revealed the important role of inductive approaches when dealing with (automated) discovery processes; (ii) in a relatively recent past, one tendency was to focus on systems' inductive capabilities in detriment (or even the explicit rejection) of the role played by the designer and/or the user along discovery processes; (iii) according to AI current tendencies, the scientific community seems to agree that a major place to human intervention should be assigned when dealing with such kind of problem; (iv) that is possibly due to the contribution of human intervention to successful discovery processes, both recent and classical ones; (v) human intervention needs to be explicitly considered in future projects, and this have been taken into account by a number of scholars who propose several guidelines after analysing successful projects.

By looking at the scientific rationale, such a human intervention combined with inductive approaches suggests us an iterative dynamics allowing scientific knowledge evolution. We strongly believe that, in such a context, the notion of contradiction would have its contribution to give, as it actually allows one to detect inadequacies between what is formalized under the form of a theory and what is represented from observation/experiment. Moreover, we also believe that such a notion has its contribution to give in domains not necessarily involved with scientific aims, i.e., formalized knowledge with no (universal) original pretension at all. As an evidence, one could look at fidal's method ( $\$ 1)$, in which new knowledge is considered only in a subjective/inter-subjective (local) spectrum.

\section{Conclusion}

In $\S 1$, we introduce an iterative and interactive method used by lawyers to render explicit the knowledge that enables an artificial agent to assist the tasks of contract analysis, verification, and construction. We also make the assumption that novice lawyers taking part in the process of knowledge explicitation learn something, thanks to the interaction both with humans and with the artificial agent. Such an assumption is firstly supported by the identification of some features previously pointed out by researchers from the Educational Community as important for learning events to occur in a Learning Environment. The results of the work carried out by real learners - as presented in $\$ 5$ - are for us an evidence (even if informal) that our initial assumption was right.

The main focus of our work is on the exam of how contradiction rationaly emerges from $y$ and simultaneously drives - the interactions between a human agent (or group) and an artificial agent both embedded in a process supposed to result in considerable intellectual gain for the human side. Concerning Human Learning, the source of general criticisms addressed to the discovery learning approach, i.e., the convergence of the learning process (often dependent on the student's autonomy degree), does not appear to us a major impeachment for Phincalculus to succeed. Taking a careful look at some of the features of Fidal's method, one might notice that something there particularly conspires in favor of convergence: the fact that knowledge built up has no universal pretension at all, it is instead emerging from a group and to (be exploited by) a 
group that intends to reach an agreement. However, a formal answer to the question "under what conditions the contradiction-driven theory construction process underlying the model actually converges" is yet to be provided. Moreover, in spite of empirical evidence of the success of Phin-calculus as a model for the design of Discovery Learning Environments, a formal experiment is also foreseen. Ongoing work include investigating how the notion of contradiction can by exploited to actually guide the theory revision process. Also, collaborative work is to be investigated as human-human communication is provided upon Phi-calculus.

\section{Acknowledgements}

The work reported in this paper was partially supported by CAPES, Brasília, Brazil, under the grant BEX 1428/98-5. Part of the work was supported by the IST Projects MKBEEM (Multilingual Knowledge Based European Electronic Market place) on eycommerce and LARFLAST (LeARning Foreign Language Scientific Terminology), on enLearning.

We would like to thank Prof. Dr. Didier Ferrier and his D.E.A. class, without whom the pragmatic work reported in the paper could not have been carried out. We would like to thank the XIV Simpósio Brasileiro de Informática na Educação organization, and committee, which has accorded us the opportunity of disseminating our work.

\section{References}

1. V. Aleven and K. R. Koedinger. Limitations of student control: Do students know when they need help? In G. Gauthier, C. Frasson, and K. VanLehn, editors, Intelligent Tutoring Systems 5th International Conference, ITS 2000 Montréal, Canada, June 2000, Lecture Notes in Computer Science, pages 292-303. Springer, 2000.

2. M. Baker, E. de Vries, K. Lund, and M. Quignard. Interactions épistémiques médiatisées par ordinateur pour l'apprentissage des sciences: bilan de recherches. In C. Desmoulins, M. Grandbastien, and J.-M. Labat, editors, Environnements Interactifs pour l'Apprentissage avec Ordinateur - EIAO, La Villette, avril 2001.

3. J. Bartasis and D. Palumbo. Theory and technology: Design consideration for hypermedia/discovery learning environments. Available on-line: http://129.7.160.115/INST5931/ Discovery_Learning.html, 1995.

4. G. S. Blair and T. Rodden. The opportunities nd challenges of CSCW. JBCS, 1(1), 1994.
5. P. Brna. Modelos de colaboração. Revista Brasileira de Informática na Educação, (3):9-16, setembro 1998.

6. J. S. Bruner. On Knowing: Essays for the Left Hand. Harvard University Press, Cambridge, Mass,

1967.

7. A. J. Cañas, M. C., and M. Arguedas. Mining the web to suggest concepts during concept mapping: Preliminary results. In Prof. Sérgio Crespo C. S. Pinto, editor, XIII Simpósio Brasileiro de Informática na Educação - SBIE 2002, pages 230-237. Unisinos, 2002.

8. S. A. Cerri. Models and systems for collaborative dialogues in distance learning. In M. F. Verdejo and S. A. Cerri, editors, Collaborative Dialogue Technologies in Distance Learning, volume 133 of ASI Series F: Computers and Systems Sciences, pages 119-125. Springer-Verlag, Berlin Heidelberg, 1994.

9. A. F. Chalmers. O que é ciência afinal? Editora Brasiliense, São Paulo, 1993.

10. E. Clément, C. Demonque, L. Hansen-L $\varphi v e$, and P. Kahn, editors. La Philosophie de A à Z. Hatier, Paris, 1994.

11. N. C. A. da Costa and J.-Y. Béziau. La logique paraconsistante. In J. Sallantin and J.-J. Szczeciniarz, editors, Le concept de preuve à la lumière de l'intelligence artificielle, pages 107-115. Puf, 1999.

12. G. M. da Nóbrega. Especificação formal de um sistema de apoio à descoberta. Master's thesis, Universidade Federal da Paraíba (UFPB), Campina Grande, Paraíba, Brasil, 1998.

13. G. M. da Nóbrega. Une approche dialectique à la formation de théories : aspects conceptuels, formels et pragmatiques dans le cadre de l'apprentissage humain. PhD thesis, LIRMM/Université Montpellier II, Montpellier (France), 2002.

14. G. M. da Nóbrega, S. A. Cerri, and J. Sallantin. DIAL: serendipitous DIAlectic Learning. In $T$. Okamoto, R. Hartley, Kinshuk, and J. P. Klus, editors, IEEE International Conference on Advanced Learning Technologies - ICALT'2001, pages 109-110, Madison, Wisconsin (USA), August 6-8 2001. IEEE Computer Society. 
15. G. M. da Nóbrega, S. A. Cerri, and J. Sallantin. On the social rational mirror: learning e-commerce in a web-served learning environment. In S. A. Cerri, G. Gouardères, and F. Paraguaçu, editors, Intelligent Tutoring Systems 6th International Conference - ITS 2002 - Biarritz (France) and San Sebastian (Spain), june 2-7, LNCS 2363, pages 41-50, Berlin Heidelberg, 2002. Springer-Verlag.

16. G. M. da Nóbrega, P. Malbos, and J. Sallantin. Modeling through human-computer interactions and mathematical discourse. In L. Magnani, N. J. Nersessian, and C. Pizzi, editors, Logical and computational aspects of model-based reasoning, volume 25 of Applied Logic Series, pages 293-311. Kluwer Academic Publishers, Dordrecht, 2002.

17. J. Dewey. Experience and Education. MacMillan, New York, 1938

18. P. Dillenbourg, M. Baker, A. Blaye, and C. O'Malley. The evolution of research on collaborative learning. In E. Spada and P. Reiman, editors, Learning in Humans and Machine: Towards an interdisciplinary learning science, pages 189-211. Elsevier, Oxford, 1996.

19. E. Ferneda. Conception d'un agent rationnel et examen de son raisonnement en géométrie. $\mathrm{PhD}$ thesis, Université de Sciences et Techniques du Languedoc - Montpellier II, Montpellier, France, 1992.

20. C. G. Hempel. Fundamentals of Concept Formation in Empirical Science, volume 2(7) of International Encyclopedia of Unified Science. University of Chicago Press, Chicago, 1952.

21. D. H. Jonassen. Objectivism versus constructivism: Do we need a new philosophical paradigm? Educational Technology Research \& Development, 39:5-14, 1991.

22. H. Jong and A. Rip. The computer revolution in science: steps towards the realization of computersupported discovery environments. Artificial Intelligence, 91:225-256, 1997.

23. I. Lakatos. Proofs and Refutations : The Logic of Mathematical Discovery. Cambridge Univ Press, 1977.

24. P. Langley. The computer-aided discovery of scientific knowledge. In First International
Conference on Discovery Science, Fukuoka (Japan), 1998. Springer.

25. P. Langley. The computational support to scientific discovery. Int. J. of Human-Computer Stud., 53:393-410, 2000.

26. P. Langley, G. L. Bradshaw, and H. A. Simon. Bacon5: The discovery of conservation laws. In IJCAI-81, 1981.

27. P. Langley, H. A. Simon, G. L. Bradshaw, and J. M. Zytkow. Scientific Discovery: Computational Explorations of the Creative Processes. The MIT Press, Cambridge, MA, 1987.

28. M. Latourrette. Acquisition de connaissances à partir d'opérateurs de généralisation : vers une unification des notions de similarité et de catégories. $\mathrm{PhD}$ thesis, Université de Sciences et Techniques du Languedoc - Montpellier II, Montpellier, France, 2001

29. D. Lecourt, editor. Dictionnaire d'histoire et philosophie des sciences. Puf, Paris, 1999.

30. L. Ledesma, A. Pérez, D. Borrajo, and L. M. Laita. A computational approach to george boole's discovery of mathematical logic. Artificial Intelligence, 91:281-307, 1997.

31. D. B. Lenat. The role of heuristics in learning by discovery. In Machine Learning: An Artificial Intelligence Approach. Morgan Kaufmann, Palo Alto (USA), 1983.

32. D. B. Lenat. Theory formation by heuristic search - the nature of heuristics ii: Background and examples. Artificial Intelligence, 21:1-2, 1983.

33. M. Liquière. Du structurel au propositionnel, une approche formelle. In CAP'01, juin, 252001. http://www.lirmm.fr/ liquiere/papiers/cap2001.pdf.

34. M. Liquière and J. Sallantin. Structural machine learning with gallois lattice and graphs. In $5^{\text {th }}$ International Conference on Machine Learning (ICML'98), pages 305-313, Madison, Wisconsin (USA), 1998.

35. A. Newell, J. C. Shaw, and H. Simon. The process of creative thinking. In H. Gruber, G. Terrel, and M. Wertheimer, editors, Contemporary Approaches To 
Creative Thinking, pages 63-119. Atherton Press, New York, 1962.

36. K. Popper. The Logic of Scientific Discovery. Routledge, 1977.

37. M. Py. Un agent rationnel pour raisonner par analogie. $\mathrm{PhD}$ thesis, Université de Sciences et Techniques du Languedoc - Montpellier II, Montpellier, France, 1992.

38. J. Sallantin. Les Agents Intelligents : Essai sur la Rationalité Des Calculs. Hermès, 1997.

39. W. Sellars. Concepts as involving laws and inconceivable without them. Philosophy of Science, 15:287-315, 1948.

40. H. Simon, R. E. Valdés-Pérez, and D. H. Sleeman. Scientific discovery and simplicity of method. Artificial Intelligence, 91:177-181, 1997. (Editorial).

41. G. L. Torres, J. M. Abe, M. L. Mucheroni, and P. E. Cruvinel, editors. Advances in Intelligent Systems and Robotics - LAPTEC 2003, volume 101 of Frontiers in Artificial Intelligence and Applications. IOS Press, Amsterdam, The Netherlands, 2003.

42. E. L. Trist. The sociotechnical perspective: the evolution of sociotechnical systems as a conceptualframework and as an action research program. In A. H. van der Ven and W. F. Joyce, editors, Perspectives on Organisation Design and Behaviour, pages 19-87. Wiley, New York, 1981.

43. R. E. Valdés-Pérez. Principles of human computer collaboration for knowledge discovery in science. Artificial Intelligence, 107(2):335-346, 1999.

44. W. R. van Joolingen. Designing for collaborative discovery learning. In G. Gauthier, C. Frasson, and K. VanLehn, editors, Intelligent Tutoring Systems, 5th International Conference, ITS 2000, Montréal, Canada, June 19-23, 2000, volume 1839 of LNCS, pages 202-211, Berlin Heidelberg, 2000. SpringerVerlag.

45. M. Weitz. Theories of concepts. A History of the Major Philosophical Tradition. Routledge, Londres/NewYork, 1988. 